\title{
GLOBAL TRUDINGER-MOSER INEQUALITY ON METRIC SPACES
}

\section{ADIMURTHI AND PRZEMYSŁAW GóRKA}

Abstract. We study Trudinger-Moser type inequalities on the entire metric measure spaces. Moreover, we give the necessary and sufficient conditions under which the Trudinger-Moser inequality holds.

Mathematics subject classification (2010): 46E35, 30L99.

Keywords and phrases: Trudinger-Moser inequality, metric measure space.

\section{REFERENCES}

[1] R. A. Adams, Sobolev spaces, Pure and Applied Mathematics, Vol. 65, Academic Press, New YorkLondon, 1975

[2] P. GóRKA, Looking for compactness in Sobolev spaces on noncompact metric spaces, forthcoming.

[3] P. Górka, T. Kostrzewa, E. G. ReYes, Sobolev spaces on locally compact abelian groups: compact embeddings and local spaces, Journal of Function Spaces, (2014), ID 404738.

[4] P. Górka, T. Kostrzewa, Sobolev Spaces on Metrizable Groups, Ann. Acad. Sci. Fenn. 40 (2015), 837-849.

[5] P. GórKa, A. Macios, The Riesz-Kolmogorov theorem on metric spaces, Miskolc Math. Notes. 15 (2014), 459-465.

[6] P. GóRKA, A. MACIOS, Almost everything you need to know about relatively compact sets in variable Lebesgue spaces, J. Funct. Anal. 269 (2015), no. 7, 1925-1949.

[7] P. GóRKA, E. G. ReYes, Sobolev spaces on locally compact abelian groups and the bosonic string equation, J. Aust. Math. Soc., 98 (2015), 39-53.

[8] P. HajŁaSZ, Sobolev spaces on an arbitrary metric space, Potential Anal. 5 (1996), 403-415.

[9] P. HAJŁASZ, Sobolev spaces on metric-measure spaces. Heat kernels and analysis on manifolds, graphs, and metric spaces, (Paris, 2002), Contemp. Math. 338 (2003), 173-218.

[10] P. HajŁasz, P. Koskela, Sobolev met Poincaré, Memoirs Amer. Math. Soc. 688 (2000), 1-101.

[11] J. Heinonen, Lectures on Analysis on Metric Spaces, Universitext 2001.

[12] J. Kinnunen, O. Martio, The Sobolev capacity on metric spaces, Ann. Acad. Sci. Fenn. Math. 21 (1996), 367-382.

[13] R. PANDA, Nontrivial solution of a quasilinear elliptic equation with critical growth in $R^{n}$, Proc. Indian Acad. Sci. Math. Sci. 105 (1995), 425-444.

[14] S. Pohozaev, The Sobolev embedding in the special case $p l=n$, Proceedings of the technical scientific conference on advances of scientific research 1964-1965, Mathematics sections, 158-170, Moscow, Energel. Inst., Moscow 1965.

[15] N. S. Trudinger, On embeddings into Orlicz spaces and some applications, J. Math. Mech. 17 (1967), 473-484.

[16] V. J. YUdovich, Some estimates with integral operators and with solutions of elliptic equations, Sov. Math. Docl. 2, (1961), 746-749.

[17] Y. YANG, Trudinger-Moser inequalities on complete noncompact Riemannian manifolds, J. Funct. Anal. 263 (2012), 1894-1938.

[18] Y. YANG, Existence of positive solutions to quasi-linear elliptic equations with exponential growth in the whole Euclidean space, J. Funct. Anal. 262 (2012), 1679-1704.

[19] Y. YANG, Trudinger-Moser inequalities on the entire Heisenberg group, Math. Nachr. 287 (2014), 1071-1080. 
[20] Y. YANG, X. Zhu, Trudinger-Moser embedding on the hyperbolic space, Abstr. Appl. Anal. (2014), Art. ID 908216, 4 pp. 